\title{
Photon, diphoton and photon+jet production measured with the ATLAS detector
}

Michal Svatos* on behalf of the ATLAS Collaboration.

Institute of Physics, v.v.i., Academy of Sciences of the Czech Republic, Prague

E-mail: michal.svatosecern.ch

\begin{abstract}
A measurement of prompt photon, diphoton and photon+jet cross-section, using data collected by the ATLAS Experiment at the LHC in the years 2010 and 2011, is presented. Isolated prompt photons provide a direct probe of short-distance physics, complementary to that provided by measurements of jets or vector-bosons. The data are sensitive to the gluon density of the proton. The inclusive prompt photon cross sections have been measured over a wide range of transverse momenta; the diphoton cross section has also been measured as a function of diphoton mass, total transverse momentum and azimuthal separation; the cross section for photons produced in association with jets is also measured. The results are compared to the predictions of next-toleading-order QCD.
\end{abstract}

XXI International Workshop on Deep-Inelastic Scattering and Related Subject -DIS2013, 22-26 April 2013

Marseilles, France

*Speaker. 


\section{Introduction}

Measurement of the production of prompt photon provides a tool for probing perturbative Quantum Chromodynamics (pQCD). Prompt photon production is sensitive to the gluon content of the proton through the process $q g \rightarrow q \gamma$. Furthermore, photons are important for many physics signatures (e.g. Higgs or graviton decays to photon pair). Additionally, the angular correlations between the photon and the jet can be used to constrain the photon fragmentation functions [1]. Term prompt photon includes both direct and fragmentation photons. Direct photons originate from the hard processes which are primarily Compton-QCD $(q g \rightarrow q \gamma)$ and quark-antiquark annihilation $(q \bar{q} \rightarrow g \gamma)$. Fragmentation photons are created by the fragmentation of the high- $p_{\mathrm{T}}$ parton.

The results presented were obtained using ATLAS data at $\sqrt{s}=7 \mathrm{TeV}$ collected at LHC during the years 2010 (luminosity of $\sim 35 \mathrm{pb}^{-1}$ ) and 2011 (luminosity of $\sim 5 \mathrm{fb}^{-1}$ ).

\section{The ATLAS detector}

The ATLAS detector [2] is a multi-purpose particle physics detector with a forward-backward symmetric cylindrical geometry and nearly $4 \pi$ coverage in solid angle. It consists of three different detector systems - the Inner Detector (ID), system of calorimeters, and the Muon spectrometer. For the photon physics, the ID and calorimeters are the most relevant subdetectors.

The photons are reconstructed from clusters of calorimeter towers. The jets are reconstructed from topological clusters built from calorimeter cells, using the anti- $k_{T}$ algorithm [3].

Events are required to have at least one reconstructed primary vertex with at least three associated tracks consistent with the beam spot position.

\section{Selection of tight isolated photon}

Only tight and isolated photons are used in these analyses. Tight selection uses several shower variables to discriminate signal from background. True prompt photons are expected to have a small hadronic leakage (the ratio between the transverse energy deposited in the first layer of the hadronic calorimeter and the transverse energy of the photon candidate) and a narrower energy profile in the electromagnetic calorimeter (based on the energy in deposited in $3 \times 7$ cells and the RMS width of the energy distribution along $\eta$ in the second layer of the electromagnetic calorimeter). Tight criteria also reject wide showers consistent with jets (using the total RMS width of the energy distribution along $\eta$ ), the ones with two separated maxima in the first layer (based on the asymmetry between the first and second maxima in the energy profile along $\eta$ and the energy difference between the second maximum and the minimum between the two maxima), and two showers merged in a wider maximum (using the fraction of the energy in seven strips centered around the first maximum that is not contained in the three core strips and the RMS width of the energy distribution computed with the three core strips). Different selections provide varying efficiencies and background rejections. The tight selection is optimised for the best background rejection.

Photons are required to be isolated both in electromagnetic and hadronic calorimeters. Photon transverse isolation energy $\left(E_{T}^{i s o}\right)$ is energy deposited inside a cone of $R=\sqrt{(\Delta \eta)^{2}+(\Delta \phi)^{2}}=0.4$, excluding contributions from $5 \times 7$ electromagnetic calorimeter cells around the photon barycenter. 
The value of cut on $E_{T}^{i s o}$ is analysis dependent.

Based on isolation and identification, photons are divided into four groups. This allows for an estimate of the number of signal photons can be obtained by a 2D sideband method.

\section{Isolated prompt photon}

The isolated prompt photon cross-section was measured using data collected in the years 2010 [4] and 2011 [5].

In the 2010 analysis, photons with $45 \mathrm{GeV}<E_{T}^{\gamma}<400$ $\mathrm{GeV}$ were sorted into four pseudorapidity bins: $\left|\eta^{\gamma}\right|<$ $0.6,0.6 \leq\left|\eta^{\gamma}\right|<1.37,1.52 \leq$ $\left|\eta^{\gamma}\right|<1.81$, and $1.81 \leq$ $\left|\eta^{\gamma}\right|<2.37$. The events were selected using a single photon trigger with a threshold of $40 \mathrm{GeV}$. The photons were tight with $E_{T}^{i s o}<3 \mathrm{GeV}$. The MC samples were simulated using Pythia and Herwig.

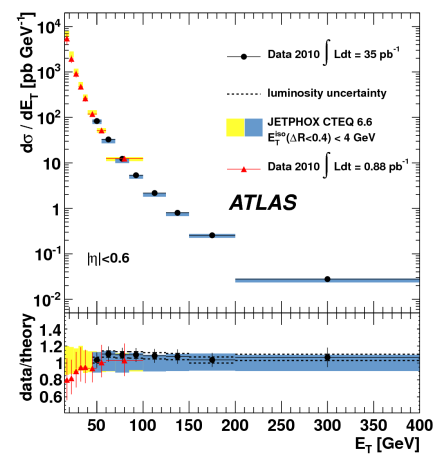

(a) prompt photon cross-section based on 2010 data, $|\eta|<0.6$ [4]

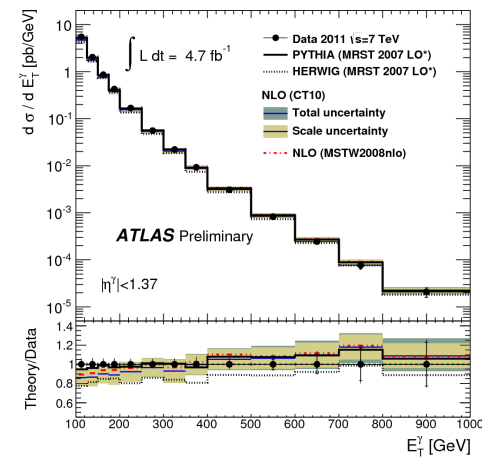

(b) prompt photon cross-section based on 2011 data, $|\eta|<1.37$ [5]

Figure 1: Measured prompt photon cross-sections in central pseudorapidity bins as a function of $E_{T}^{\gamma}$

The NLO prediction was obtained with JETPHOX.

The cross-section for the most central bin is shown in Figure 1a. It also contains results from a previous analysis [6] using data starting at $E_{T}^{\gamma}=15 \mathrm{GeV}$. Both statistical and systematic uncertainties are included in the error bars. The NLO prediction describes data - except the low $E_{T}^{\gamma}$ region. The underestimation of uncertainty associated with the NLO predictions is caused by the contribution of a fragmentation component.

In the analysis of 2011 data, photons were sorted into two pseudorapidity bins: $\left|\eta^{\gamma}\right|<1.37$ and $1.52 \leq\left|\eta^{\gamma}\right|<2.37 . E_{T}^{\gamma}$ was in the region $100-1000 \mathrm{GeV}$. Single photon trigger with the threshold of $80 \mathrm{GeV}$ was used. The photons were tight with $E_{T}^{i s o}<7 \mathrm{GeV}$. The simulated samples were created using Pythia and HeRwig. The NLO prediction was obtained with JetPhOX.

A result of 2011 data analysis for the most central bin is shown in Figure 1b. The NLO prediction is in agreement with the data. PythiA describes data well, HeRWIG values lie below the data.

\section{Diphoton}

The isolated photon pair cross-section was measured using data collected in 2011 [7]. Photons were sorted into two pseudorapidity bins: $\left|\eta^{\gamma}\right|<1.37$ and $1.52 \leq\left|\eta^{\gamma}\right|<2.37$. The leading photon was required to have $E_{T 1}^{\gamma}>25 \mathrm{GeV}$, the subleading one $E_{T 2}^{\gamma}>22 \mathrm{GeV}$. The angular separation between the two photons was $\Delta R>0.4$. The events were selected using a diphoton trigger with threshold of $20 \mathrm{GeV}$. The photons were tight with $-4 \mathrm{GeV}<E_{T}^{\text {iso }}<4 \mathrm{GeV}$ (because 2D template fit, performed as a cross-check, starts at $-4 \mathrm{GeV}$ ). The predictions were provided by PYTHIA and SHERPA at LO, by DiPHOX complemented by GAMMA2MC at NLO, and by $2 \gamma$ NNLO at NNLO. 


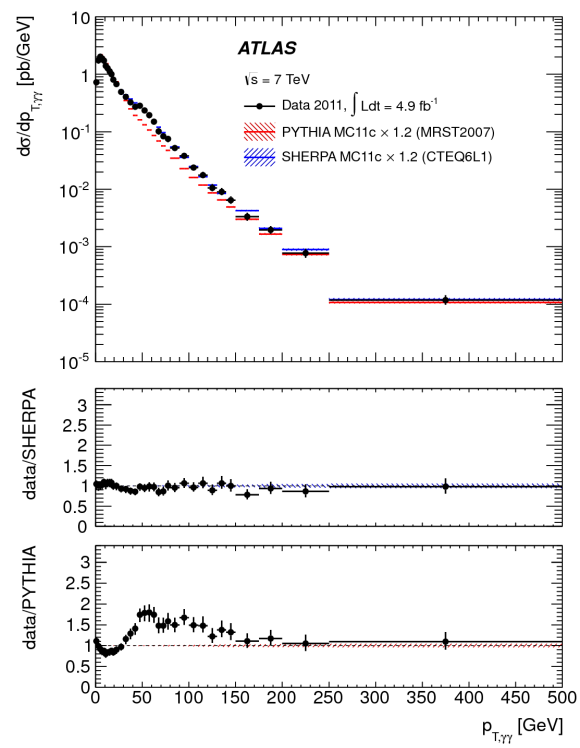

(a) diphoton cross-section and comparison with LO predictions [7]
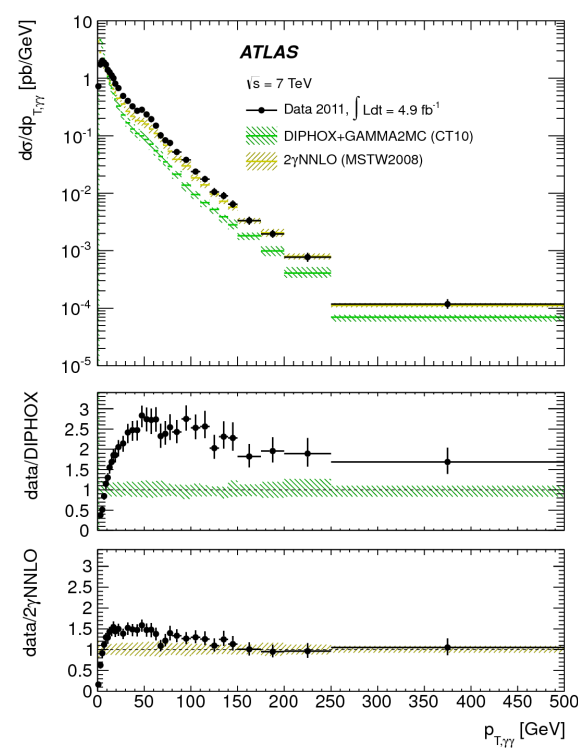

(b) diphoton cross-section and comparison with NLO and NNLO predictions [7]

Figure 2: Measured diphoton cross-sections as a function of $p_{T}^{\gamma \gamma}$ and comparisons with LO, NLO, and NNLO predictions

The results of the diphoton analysis are shown in the Figures $2 \mathrm{a}$ and $2 \mathrm{~b}$. Error bands include only statistical uncertainty for the LO comparison. The generators mostly underestimate the data because of missing higher orders contributions. SHERPA is closer to data than PYTHIA. For NLO and NNLO, statistical, PDF and scale uncertainties are included in the error band. The NLO prediction underestimates the data because of missing NNLO corrections. The NNLO prediction underestimates the data in the regions populated by the contribution from fragmentation because $2 \gamma$ NNLO only provides a description of the direct component. $2 \gamma \mathrm{NNLO}$ agrees with data better than DiPHOX+GAMMA2MC.

\section{Photon+jet}

There are two measurements of photon+jet cross-section using 2010 data: the first one focusing on the basic cross-section measurement [8] and the second one on the photon+jet dynamics [9]. In the first analysis, photons with $E_{T}^{\gamma}>25 \mathrm{GeV}$ were measured in pseudorapidity range $\left|\eta^{\gamma}\right|<1.37$. The events were selected using a single photon trigger with a threshold of $40 \mathrm{GeV}(20 \mathrm{GeV})$ for $E_{T}^{\gamma}>45 \mathrm{GeV}\left(E_{T}^{\gamma}>25 \mathrm{GeV}\right)$. The photons were tight with $E_{T}^{i s o}<3 \mathrm{GeV}$. The jets were from the region $\left|y^{\text {jet }}\right|<4$.4. The minimum separation between leading jet and photon was $\Delta R>1.0$. Jets were defined using anti- $k_{t}$ algorithm with $\mathrm{R}=0.4$. Simulated samples were created using PYTHIA, HERWIG + JiMmY and JETPHOX for both analyses, SHERPA was used only in the first one.

A resulting basic cross-section is shown in Figure 3a. It was measured in three jet rapidity bins: $\left|y^{j e t}\right|<1.2,1.2<\left|y^{j e t}\right|<2.8$, and $2.8\left|y^{j e t}\right|<4.4$ for two configurations: $\eta^{\gamma} y^{\text {jet }} \geq 0$ and $\eta^{\gamma} y^{\text {jet }}<0$. The errors in the figures include both statistical and systematic uncertainties. NLO prediction is in agreement with the measured cross sections.

The analysis of the dynamics investigated the cross-section as a function of several variables. The 


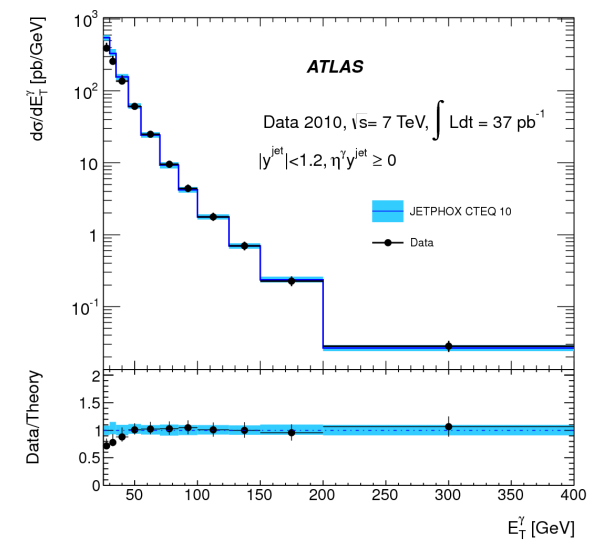

(a) Photon+jet cross-sections as a function of $E_{T}^{\gamma}$ for $\left|y^{j e t}\right|<1.2$ and $\eta^{\gamma} y^{j e t} \geq 0$ [8]

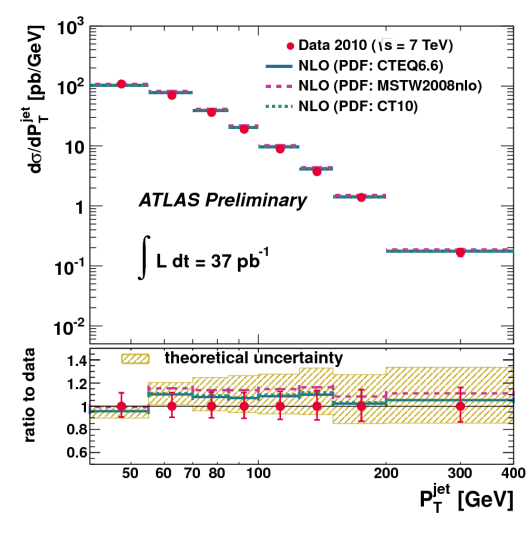

(b) Photon+jet cross-sections as a function of $p_{T}^{\text {jet }}[9]$

Figure 3: Measured photon+jet cross-sections as a function of $E_{T}^{\gamma}$ and $p_{T}^{\text {jet }}$

phase space of events was as follows: $E_{T}^{\gamma}>45 \mathrm{GeV},\left|\eta^{\gamma}\right|<1.37,1.52 \leq\left|\eta^{\gamma}\right|<2.37, P_{T}^{\text {jet }}>40$ $\mathrm{GeV},\left|\eta^{j e t}\right|<2.37, \Delta R_{\gamma j}^{2}>1$. Jets were defined using anti- $k_{t}$ algorithm with $\mathrm{R}=0.6$. The photons were tight with $E_{T}^{\text {iso }}<3 \mathrm{GeV}$.

A measured cross-section is shown in Figure 3b. NLO prediction is in fair agreement with data.

\section{Summary and conclusion}

A measurement of prompt photon, diphoton and photon+jet cross-section, using data at $\sqrt{s}=7$ $\mathrm{TeV}$ from the years 2010 and 2011, is presented. The prompt photon production is in good agreement with predictions except for very low values of $E_{T}^{\gamma}$ corresponding to small values of $x_{T}$. NLO prediction is less accurate in this region, PYTHIA describes data well and HERWIG values lie below the data. For diphoton production, predictions underestimate data due to the various reasons: PyTHIA and SHERPA because of missing higher-order contributions, DiPHOX+GAMMA2MC because of missing NNLO contributions, and $2 \gamma$ NNLO because of missing fragmentation component. Photon+jet production is in fair agreement with both LO and NLO predictions.

\section{References}

[1] Z. Belghobsi, M. Fontannaz, J. -P. .Guillet, G. Heinrich, E. Pilon and M. Werlen, Phys. Rev. D 79, 114024 (2009) [arXiv:0903.4834 [hep-ph]].

[2] ATLAS Collaboration, JINST 3, S08003 (2008).

[3] M. Cacciari, G. P. Salam and G. Soyez, JHEP 0804, 063 (2008) [arXiv:0802.1189 [hep-ph]].

[4] ATLAS Collaboration, Phys. Lett. B 706, 150 (2011) [arXiv:1108.0253 [hep-ex]].

[5] ATLAS Collaboration, ATLAS-CONF-2013-022, https : / / cds . cern. ch/record/1525723.

[6] ATLAS Collaboration, Phys. Rev. D 83, 052005 (2011) [arXiv:1012.4389 [hep-ex]].

[7] ATLAS Collaboration, JHEP 1301 (2013) 086 [arXiv:1211.1913 [hep-ex]].

[8] ATLAS Collaboration, Phys. Rev. D 85, 092014 (2012) [arXiv:1203.3161 [hep-ex]].

[9] ATLAS Collaboration, ATLAS-CONF-2013-023, https : / cds.cern. ch/record/1525728 